\title{
A 2.4 GHz Cross Rhombic Antenna for a Cube Satellite Application
}

\author{
Jorge Sosa-Pedroza, Sergio Peña Ruiz, and Fabiola Martínez-Zúñiga \\ Unidad Profesional Adolfo López Mateos, Escuela Superior de Ingeniería Mecánica y Eléctrica, Instituto Politécnico Nacional, \\ Edificio Z-4, 3er. Piso, Colonia Lindavista, 07738 Mexico, DF, Mexico
}

Correspondence should be addressed to Sergio Peña Ruiz; srgpr13@gmail.com

Received 26 August 2013; Revised 22 December 2013; Accepted 23 December 2013; Published 19 February 2014

Academic Editor: Xiao Ping Chen

Copyright (C) 2014 Jorge Sosa-Pedroza et al. This is an open access article distributed under the Creative Commons Attribution License, which permits unrestricted use, distribution, and reproduction in any medium, provided the original work is properly cited.

\begin{abstract}
We present design and construction results of a $2.4 \mathrm{Ghz}$ cross rhombic antenna to be used in a cubesat. Computational design agrees with experimental results after its construction. cross rhombic antenna is a novel planar structure of our own design, presenting circular polarization and medium gain; it is built over a RF60A substrate with $\varepsilon_{r}=6.15$, decreasing its size to fit the required dimensions of satellite. A special characteristic of this design is the enhancing of operational bandwidth using a technique we have been studying, related to softening the structures with sharp edges. Results show applicability and success of our technique.
\end{abstract}

\section{Introduction}

A cube satellite is a very small spacecraft, usually having an area as much as $10 \mathrm{~cm}^{2}$ and no much longer than $30 \mathrm{~cm}$; its weight should be less than $1 \mathrm{Kg}$. Most of these small satellites use commercial electronic components. California Polytechnic State University and Stanford University issued the CubeSat specifications in 1999, helping universities to develop the science and space exploration; these specifications are followed by amateur radio satellite builders. It is easy to imagine that the $10 \mathrm{~cm}^{2}$ satellite area requires an antenna to fit those dimensions. We have been working with planar antennas for long time, developing, designing and constructing our own structures [1]. Some of them have been proposed to be used in different applications. We present in this paper a study of one of those structures: the Cross Rhombic Antenna (CRA). Actually, there are many kinds of planar antennas having different forms. Planar antennas are very popular considering their small dimensions and low cost making them ideal for many applications where small dimensions are needed, as in airplanes, spacecraft, and fixed terrestrial communications taking a great importance in last generation of these systems. Some characteristics to be considered for the antenna design are the following:

(i) high reliability; (ii) small size;

(iii) low weight;

(iv) high efficiency;

(v) low cost.

The cubesat dimensions impose as well antenna dimensions, keeping the best behavior. As we show in Section 3, analysis was done in order to get the best behavior for a $10 \mathrm{~cm}^{2}$ of satellite face. CRA is proposed to be used in Sensat, a cube satellite, working at $2.4 \mathrm{GHz}$. Sensat is a small educational satellite projected with optical and microwave payloads, developed by several Mexican universities.

\section{The Cross Rhombic Antenna}

CRA belongs to the family of traveling wave antennas, with circular polarization, medium gain, and a directional field pattern. Antenna is fed in one end and loaded in the other one, to meet expected behavior. The parametric analysis we present takes in account different characteristics of the antenna such as: microstrip width, load impedance, type of substrate and substrate thickness as well as softening of sharp corners. Antenna behavior is related to its gain, field pattern, axial ratio, and $50 \Omega$ input coupling; frequency design was 


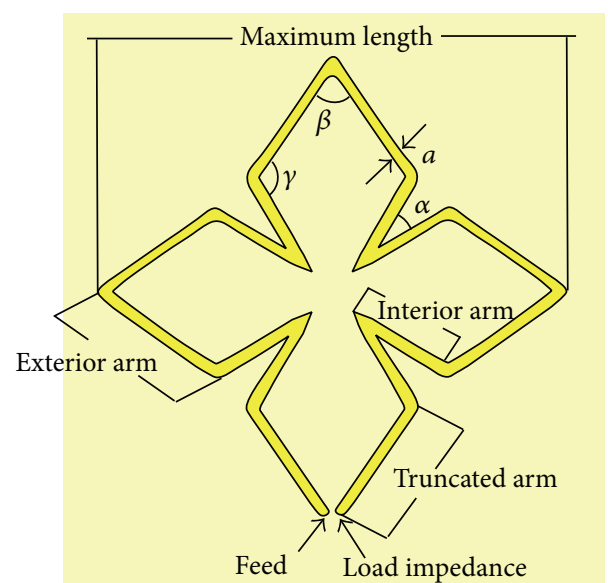

FIgURE 1: Cross rhombic antenna.

2.4 GHz and we use antenna, Computer Simulation Technology (CST) simulator. Modifying structure characteristics using a RF60A substrate with $\varepsilon_{r}=6.15$, we select the best one to construct an antenna prototype to compare it with simulation results. CRA is an evolution of the antenna proposed by Roederer in 1990 [2]. Antenna is a rhomb shape microstrip over a ground plane as shown in Figure 1. Circular right or left polarization depends on feed and load position. Load impedance could be any value but we have noticed that the best results are obtained using short circuit, open circuit or $50 \Omega$.

\section{Parametric Analysis Using RF60A Substrate}

In this section, simulation results are presented, modifying physical characteristics of the RF60A substrate structure having $\varepsilon_{r}=6.15$ and $\lambda=0.0504 \mathrm{~m}$ at $2.4 \mathrm{GHz}$. At this frequency, substrate width is $h=\lambda / 11$. Original design considers the maximum dimension (Figure 1 ) as $1.67 \lambda$ or $8.4 \mathrm{~cm}$, matching the $10 \mathrm{~cm}^{2}$ area of cube satellite. Physical parameters considered were as follows: microstrip width, load impedance, substrate thickness, and softening of sharp corners.

3.1. Microstrip Line Width. First case of analysis was the microstrip line widths. Table 1 shows the relationship between gain and line width, for the three selected load impedances, while Figures 2, 3, and 4 show the related microstrip line width to return loss parameter for each load.

As seen in Table 1, the best gain results are obtained for $a_{3}=0.033 \lambda=1.7 \mathrm{~mm}$ with open circuit load, but we choose $a_{5}=0.041 \lambda=2.1 \mathrm{~mm}$, considering the better coupling results of Figure 7; however, as seen in Figure 17, there is a better coupling for $a_{6}$ and $a_{7}$ but at higher frequencies. Figure 3 shows a better coupling with thicker lines for the $50 \Omega$ load, but the gain is lower, as seen in Table 1. For short circuit load, gain is similar to that of open circuit load and better coupling but at higher frequency; to reduce coupling frequency is necessary to increase antenna dimensions which is not desirable. Considering these results, we choose $2.1 \mathrm{~mm}$
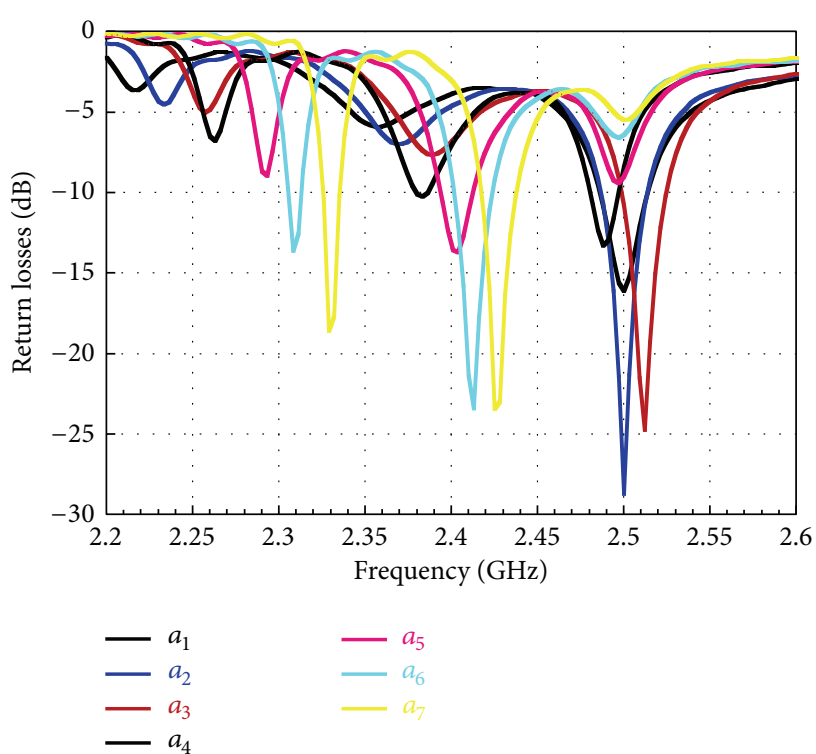

FIgURE 2: Return losses (open load line analysis).

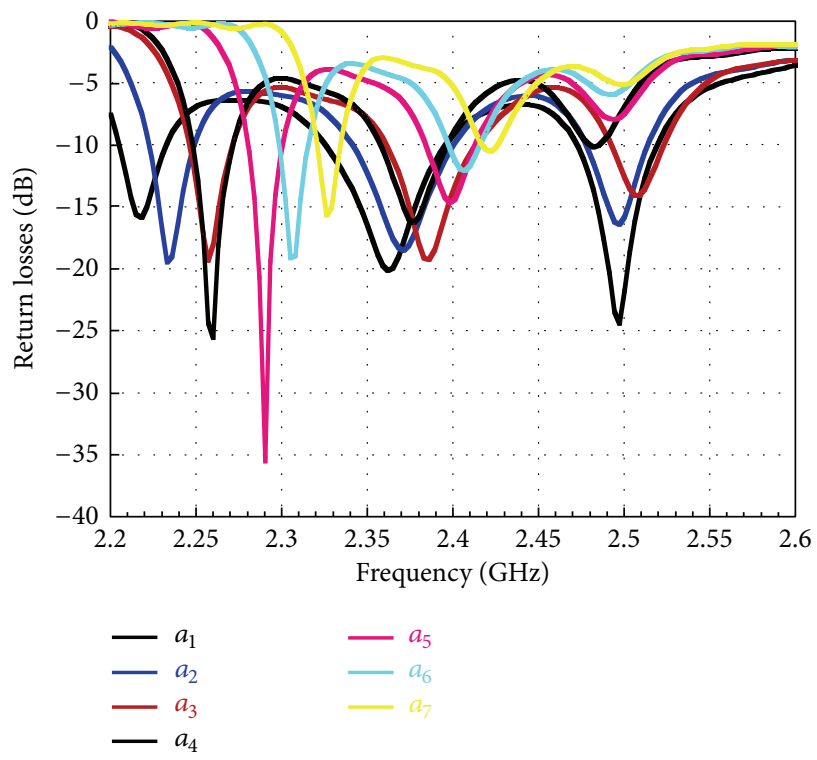

Figure 3: Return losses ( $50 \Omega$ load line analysis).

for microstrip line width and open circuit load. Figure 5 shows field patterns $\left(\phi=90^{\circ}\right.$ and $\left.\phi=180^{\circ}\right)$ and we can see similarity between all of them, and then they are not a parameter to be used for comparison.

\section{Further Size Reduction}

We tried a further size reduction of RCA, moving corners to the antenna center, but with little changes in microstrip lines width, as shown in Figure 6. Table 2 shows the final dimensions. To define the new microstrip width, we analyze the effects on gain and coupling; results are shown in Table 3 and Figure 7. Figure 8 depicts the different field patterns for each microstrip width. 


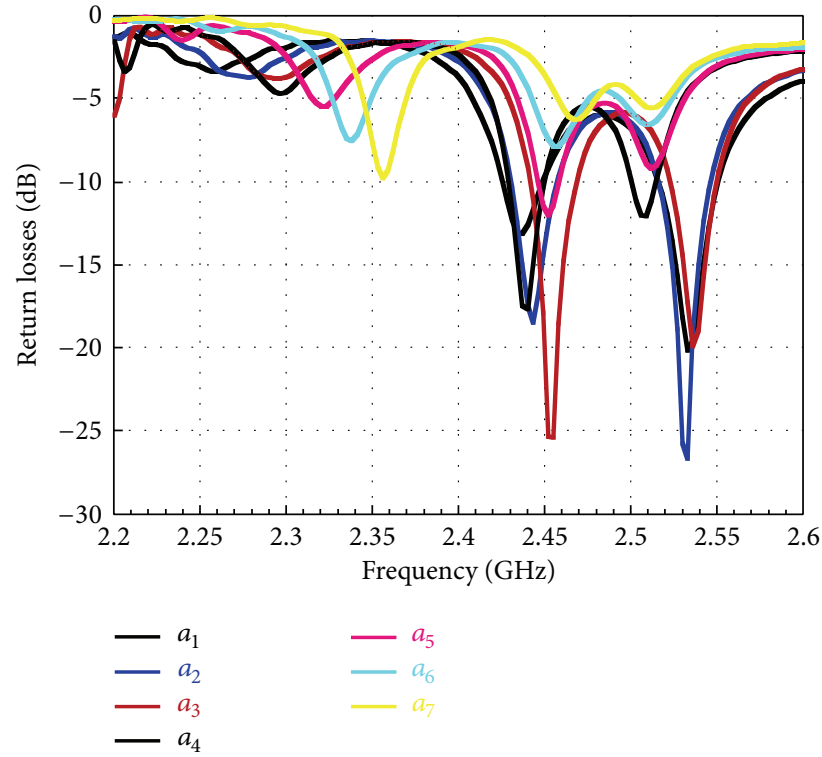

FIGURE 4: Return losses (short load line analysis).

TABLE 1: Line width analysis (RF60A).

\begin{tabular}{lcccc}
\hline $\begin{array}{l}\text { Width } \\
(\mathrm{mm})\end{array}$ & $\begin{array}{c}\text { Width } \\
(\lambda)\end{array}$ & $\begin{array}{c}\text { Gain open } \\
(\mathrm{dBi})\end{array}$ & $\begin{array}{c}\text { Gain } 50 \Omega \\
(\mathrm{dBi})\end{array}$ & $\begin{array}{c}\text { Gain short } \\
(\mathrm{dBi})\end{array}$ \\
\hline$a_{1}=1.3$ & $0.025 \lambda$ & 8.8 & 5.8 & 8.8 \\
$a_{2}=1.5$ & $0.029 \lambda$ & 8.8 & 5.8 & 8.7 \\
$a_{3}=1.7$ & $0.033 \lambda$ & 8.9 & 5.7 & 8.7 \\
$a_{4}=1.9$ & $0.037 \lambda$ & 8.8 & 6.5 & 8.6 \\
$a_{5}=2.1$ & $0.041 \lambda$ & 8.8 & 6.1 & 8.5 \\
$a_{6}=2.3$ & $0.045 \lambda$ & 8.6 & 6.0 & 8.4 \\
$a_{7}=2.5$ & $0.049 \lambda$ & 8.3 & 5.6 & 8.0 \\
\hline
\end{tabular}

TABLE 2: Antenna dimensions (RF60A).

\begin{tabular}{lcc}
\hline & Original & Modified \\
\hline Maximum length & $1.67 \lambda$ & $1.62 \lambda$ \\
Interior arm & $0.32 \lambda$ & $0.31 \lambda$ \\
Exterior arm & $0.51 \lambda$ & $0.49 \lambda$ \\
Truncated arm & $0.46 \lambda$ & $0.45 \lambda$ \\
Angle between interior arms $(\alpha)$ & $31.42^{\circ}$ & $31.42^{\circ}$ \\
Angle between exterior arms $(\beta)$ & $69.99^{\circ}$ & $69.99^{\circ}$ \\
Angle interior-exterior arms $(\gamma)$ & $115.71^{\circ}$ & $115.71^{\circ}$ \\
\hline
\end{tabular}

\section{Substrate Thickness}

Although we can make any change in dimensional antenna characteristics in simulation process, a limitation arises if we want to construct it, because commercial substrates have defined thickness dimensions; then we change that dimension in order to know its effects on radiation parameters, taking special attention in results with the materials on hand. Using the best former results, simulation was made for different substrate thicknesses, as Table 4, shows with gain results for each one. Figure 9 depicts the coupling results.
TABLE 3: Antenna gain line analysis.

\begin{tabular}{lc}
\hline Width $(\mathrm{mm})$ & Gain $(\mathrm{dBi})$ \\
\hline$a_{1}=1.5$ & 8.3 \\
$a_{2}=1.6$ & 8.3 \\
$a_{3}=1.7$ & 8.3 \\
$a_{4}=1.8$ & 8.3 \\
$a_{5}=1.9$ & 8.2 \\
$a_{6}=2.0$ & 8.2 \\
$a_{7}=2.1$ & 8.1 \\
\hline
\end{tabular}

TABLE 4: Antenna gain substrate thickness analysis.

\begin{tabular}{lc}
\hline Thickness $(\mathrm{mm})$ & Gain open $(\mathrm{dBi})$ \\
\hline$h_{1}(\lambda / 5)=10$ & 5.4 \\
$h_{2}(\lambda / 6)=8.4$ & 5.7 \\
$h_{3}(\lambda / 7)=7.2$ & 6.8 \\
$h_{4}(\lambda / 8)=6.3$ & 7.7 \\
$h_{5}(\lambda / 9)=5.6$ & 8.1 \\
$h_{6}(\lambda / 10)=5.04$ & 8.1 \\
$h_{7}(\lambda / 11)=4.58$ & 8.2 \\
$h_{8}(\lambda / 12)=4.2$ & 8.3 \\
$h_{9}(\lambda / 13)=3.8$ & 8.3 \\
$h_{10}(\lambda / 14)=3.6$ & 8.3
\end{tabular}

Figure 9 depicts the coupling results, as seen $h=\lambda / 11$ has again the best coupling results, but the commercial thickness of substrate we have is $h=\lambda / 9=5.6 \mathrm{~mm}$. We keep the analysis of both the thickness substrates, in the following paragraphs, as a way of comparison.

\section{Softening Sharp Edges to Enhance Bandwidth}

It can be noticed in $S_{11}$ parameters of former figures that curves are very sharp with small bandwidth, making antenna design and construction with a high dependence on frequency; repeatability could be a problem considering high dependency of antenna parameters of structure dimensions. To avoid this problem, we propose to increase bandwidth softening antenna corners, making it less dependent on frequency $[3,4]$; to set those changes we selected the best parameters of the analysis, that is, strip line width $a=$ $2.0 \mathrm{~mm}$, substrate thickness $h=\lambda / 11$, and open circuit load; antenna modification is shown in Figure 10.

We noticed a small change in frequency coupling after softening edges; to solve this problem, all dimensions were modified and a new substrate thickness analysis was done in order to get a new optimum value for $h$ at the $2.4 \mathrm{GHz}$ resonant frequency; simulation results are presented in Table 5 and Figure 11. Good coupling for $h_{4}=\lambda / 8$ and $h_{5}=\lambda / 9$ is noticed, and the last one is precisely the thickness we have to construct; Figures 12 and 13 show field patterns for those thicknesses.

Final optimized dimensions are presented in Table 6. An antenna gain of $8.1 \mathrm{dBi}$ is noticed. Figure 14 depicts the $S_{11}$ 


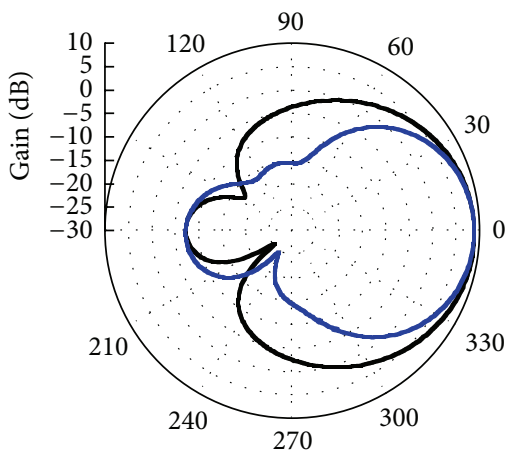

- $\phi=180^{\circ}$

$-\phi=90^{\circ}$

(a)

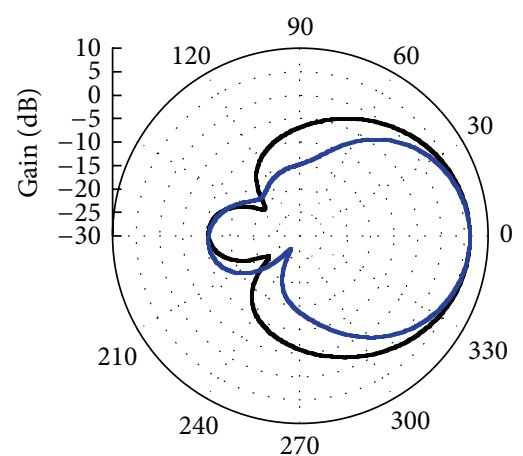

$\begin{aligned} \phi & =180 \\ -\phi & =90^{\circ}\end{aligned}$

(b)

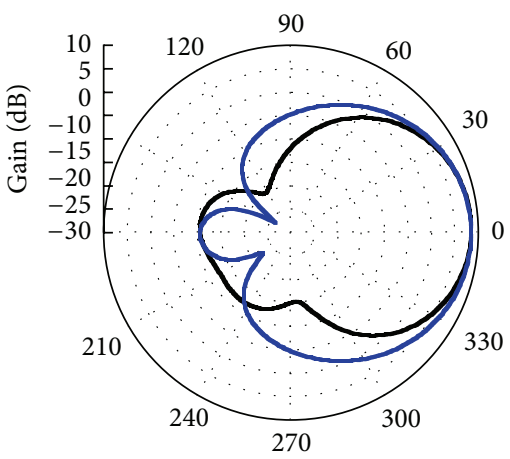

- $\phi=180^{\circ}$

(c)

FIGURE 5: Field patterns. (a) Open, (b) $50 \Omega$, and (c) short.

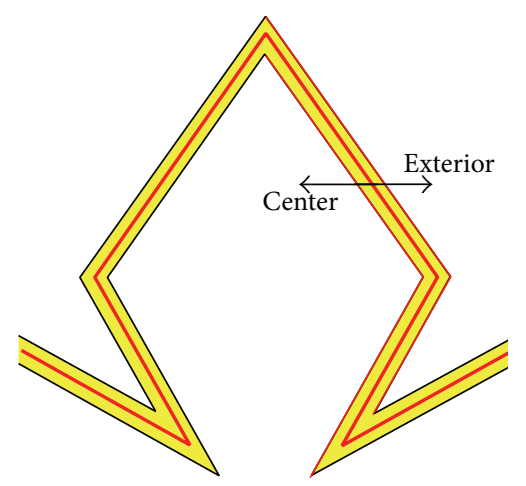

Figure 6: CRA modification.

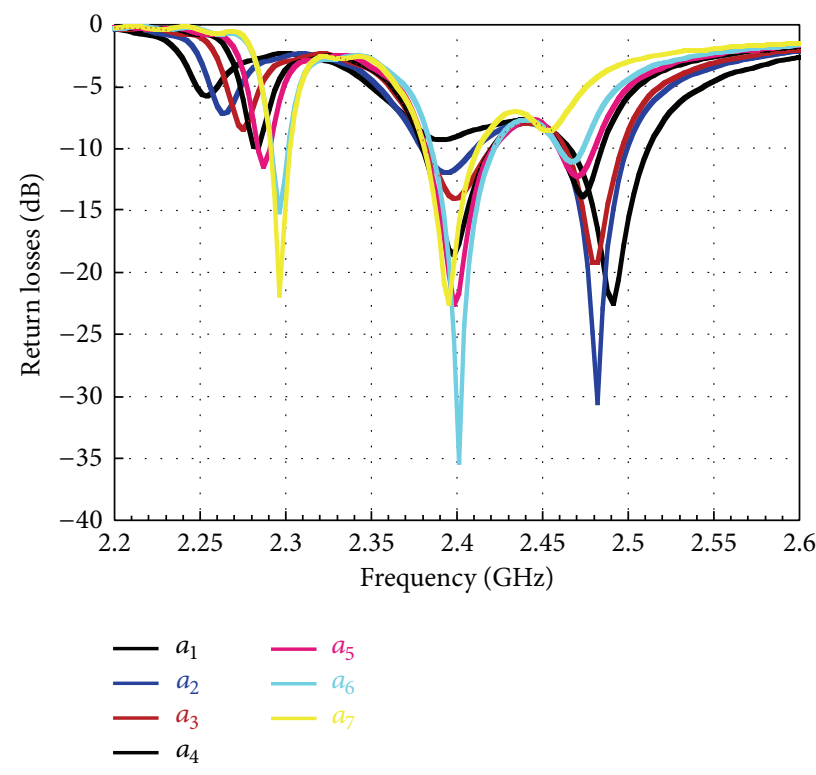

FIgURE 7: Return losses for different line widths. 


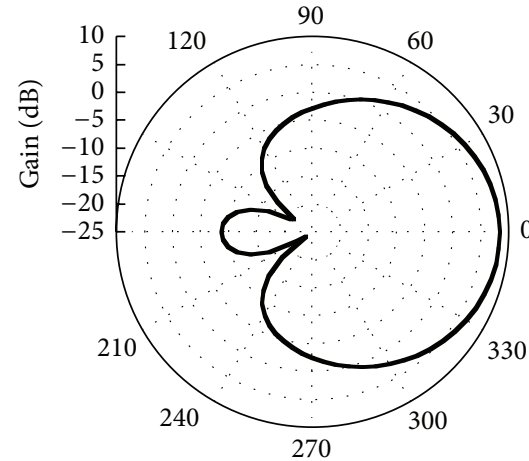

(a)

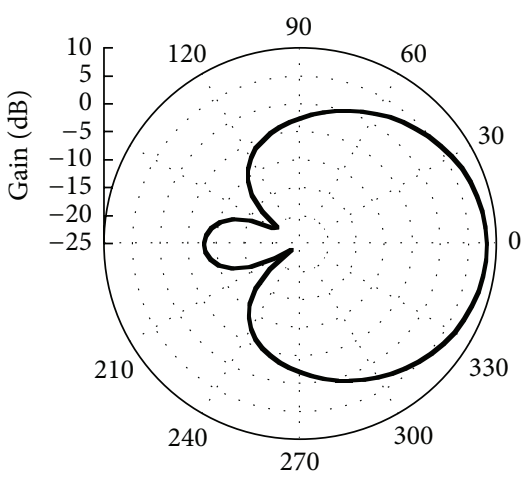

(d)

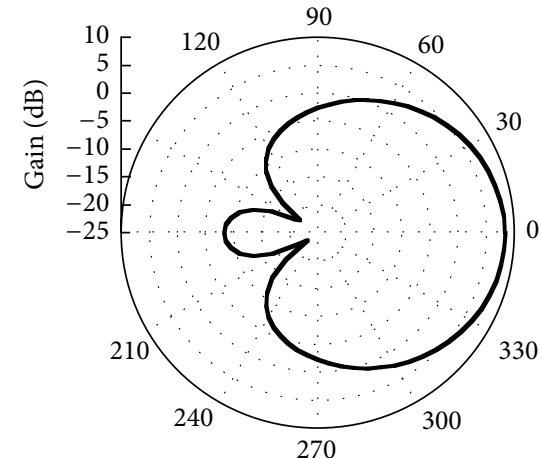

(b)

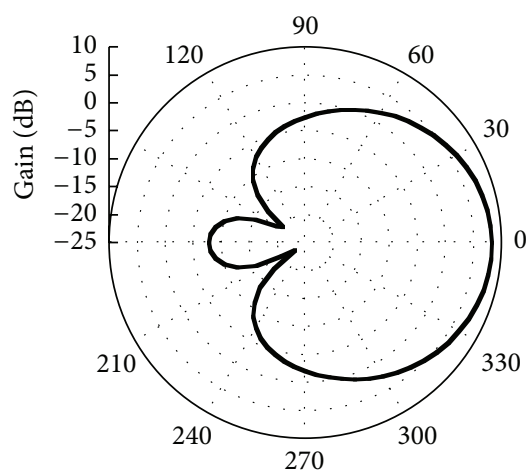

(e)

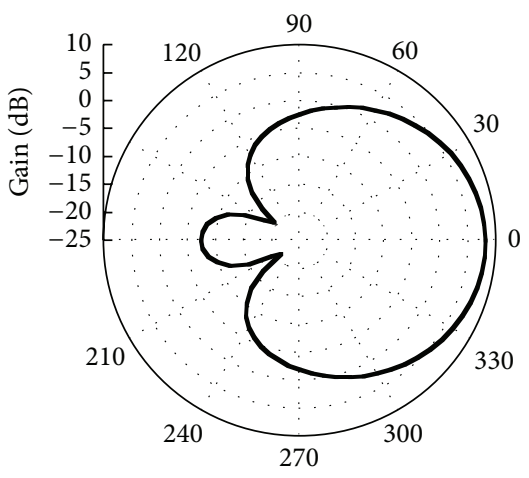

(g)

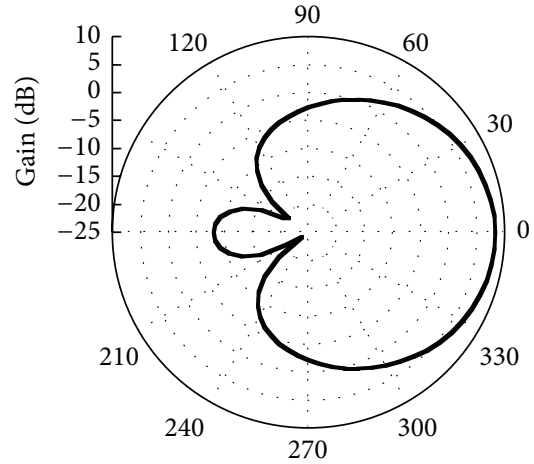

(c)

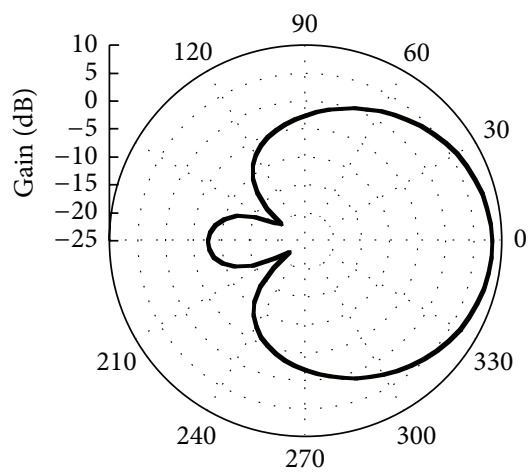

(f)
TABLE 5: Antenna gain substrate thickness analysis.

\begin{tabular}{lc}
\hline$h$ & Gain $(\mathrm{dBi})$ \\
\hline$a=\lambda / 6$ & 6.1 \\
$b=\lambda / 7$ & 7.4 \\
$c=\lambda / 8$ & 7.8 \\
$d=\lambda / 9$ & 8.1 \\
$e=\lambda / 10$ & 8.2 \\
$f=\lambda / 11$ & 8.1 \\
$g=\lambda / 12$ & 8.2
\end{tabular}

TABLE 6: Optimized antenna dimensions.

\begin{tabular}{lcc}
\hline & Optimized & Original \\
\hline Total diameter & $1.66 \lambda$ & $1.62 \lambda$ \\
Interior arm & $0.32 \lambda$ & $0.31 \lambda$ \\
Exterior arm & $0.50 \lambda$ & $0.49 \lambda$ \\
Truncated arm & $0.46 \lambda$ & $0.45 \lambda$ \\
Line width $(a)$ & $0.042 \lambda$ & $0.039 \lambda$ \\
Angle between interior arms $(\alpha)$ & $27.19^{\circ}$ & $31.42^{\circ}$ \\
Angle between exterior arms $(\beta)$ & $69.99^{\circ}$ & $69.99^{\circ}$ \\
Angle interior-exterior arms $(\gamma)$ & $115.71^{\circ}$ & $115.71^{\circ}$ \\
\hline
\end{tabular}




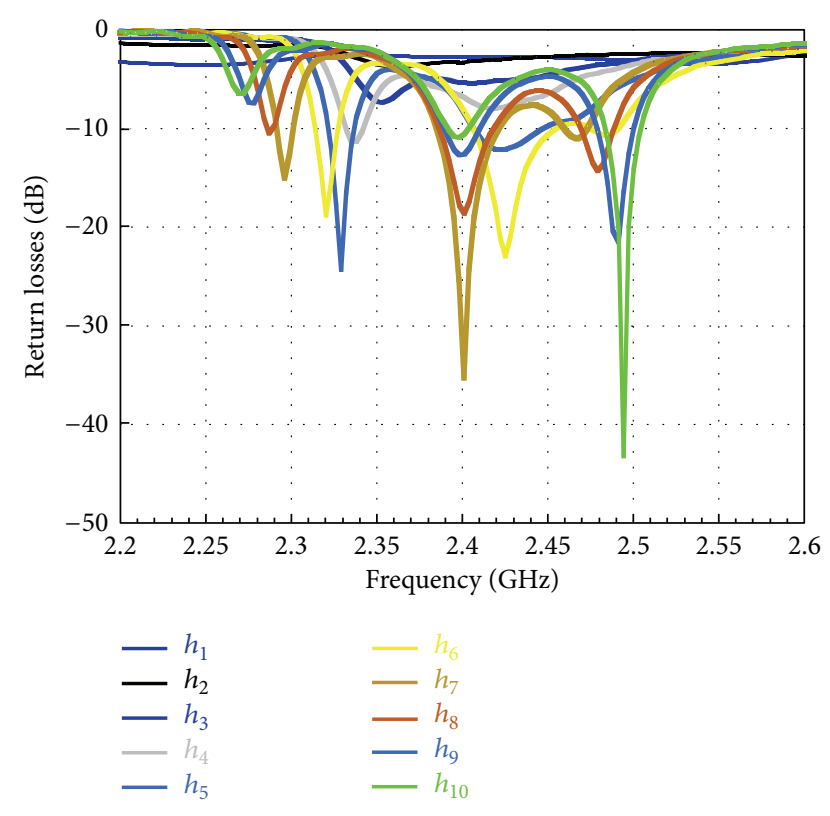

FIGURE 9: Return losses for different substrate thicknesses.

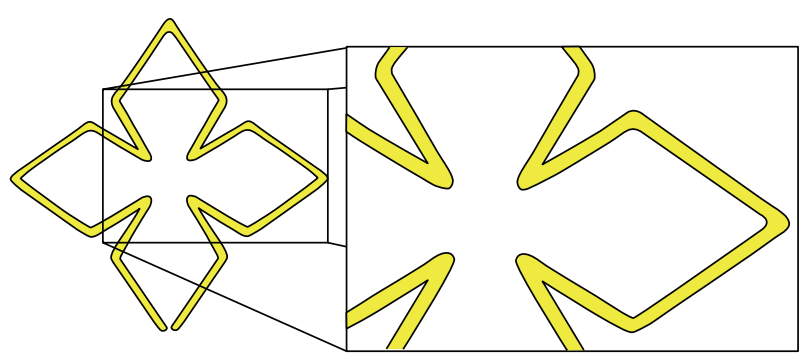

FIGURE 10: Cross rhombic antenna with softened edges.

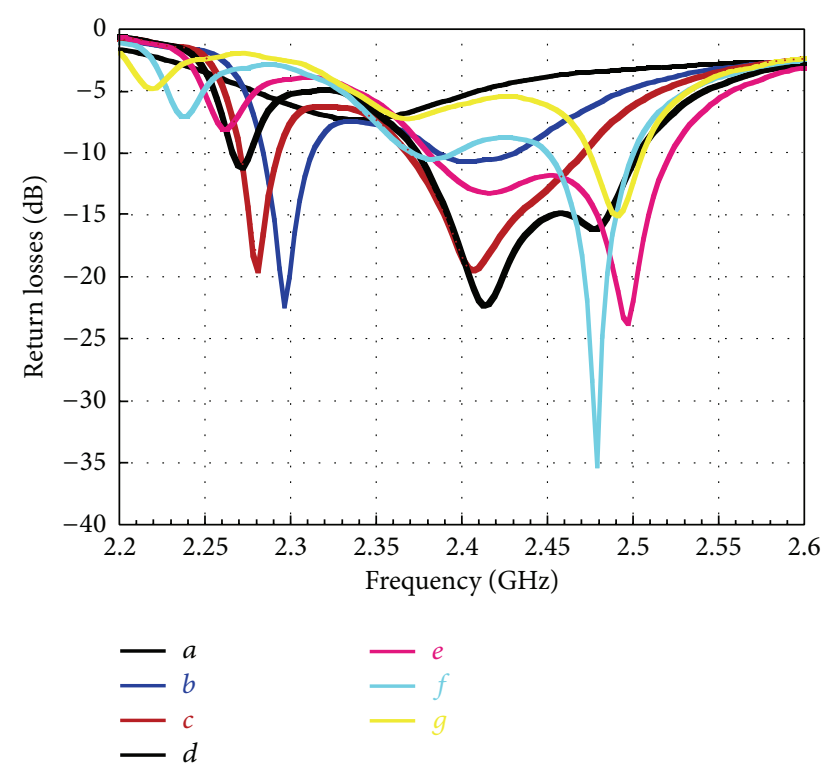

FIGURE 11: Return losses for different substrate thicknesses.

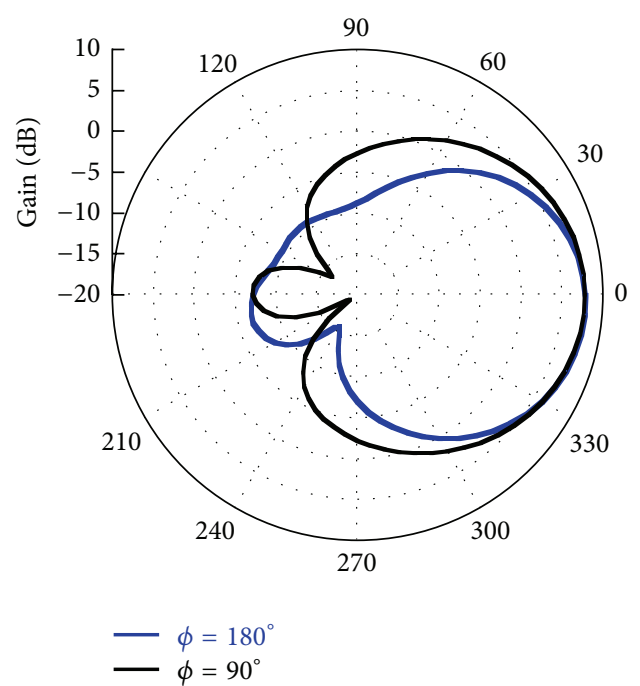

FIGURE 12: Field pattern for $h=\lambda / 8$.

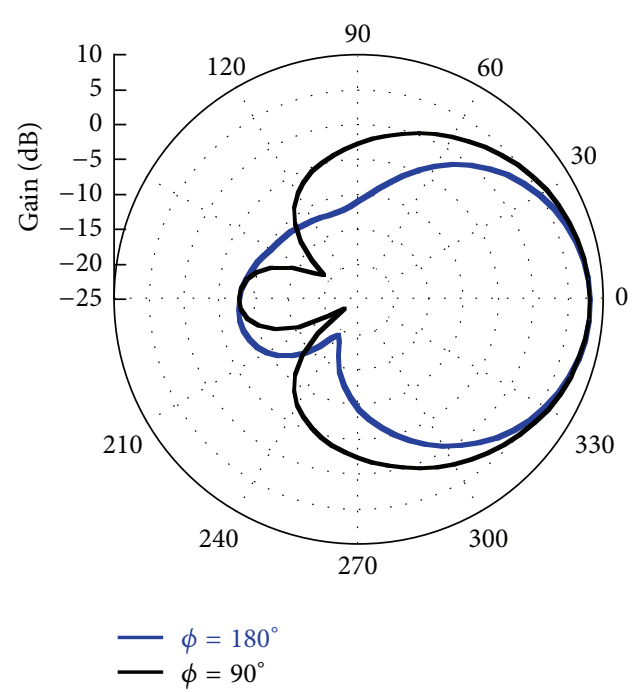

FIGURE 13: Field pattern for $h=\lambda / 9$.

parameter response comparison, with and without softening, for $h=\lambda / 9$; as it is seen, there are a higher bandwidth and a better response for the optimized antenna. As a way of comparison with other kind of antennas, the rectangular microstrip slot patch presented in [5] has narrower bandwidth and less gain.

\section{Construction and Comparison}

Two different antennas were constructed, one of them with sharp edges and the other with softened edges. Both antennas are shown in Figure 15, using RF60A substrate with $\varepsilon_{r}=6.15$ $(\lambda=5 \mathrm{~cm}$ at $2.4 \mathrm{GHz})$ and thicknesses of $h_{5}=\lambda / 9$.

Measurements were done with an Anritsu MS4624B Vector Network Measurement System. Figure 16 shows $S_{11}$ comparison between simulation and construction at $h_{5}=$ $\lambda / 9$ (real) and $h_{7}=\lambda / 11$ (optimal); as seen, there is very 


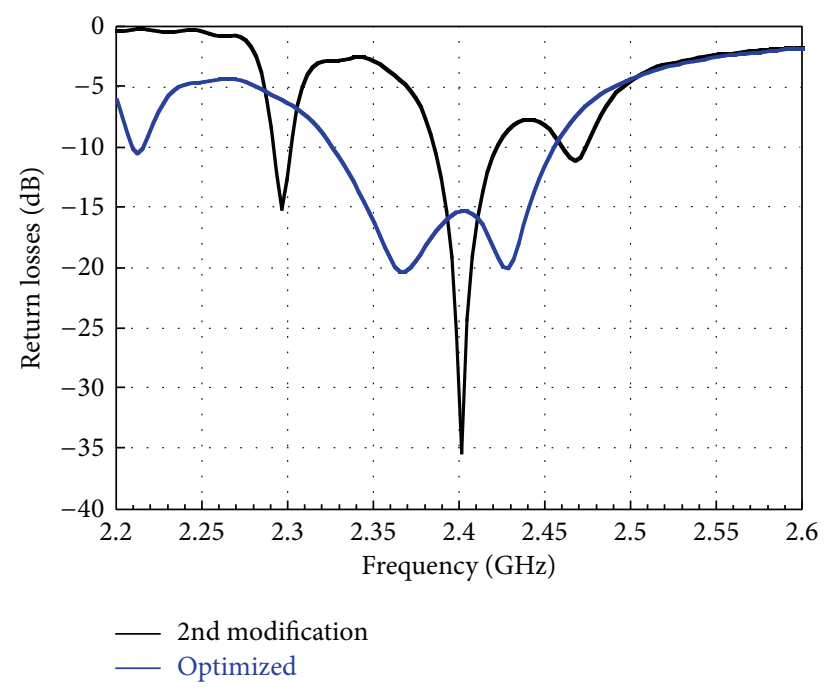

FIGURE 14: Comparison between sharp and softened ends antennas.

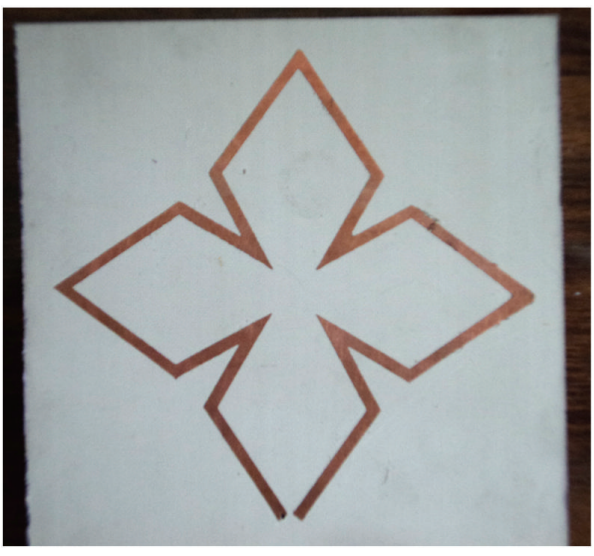

(a)

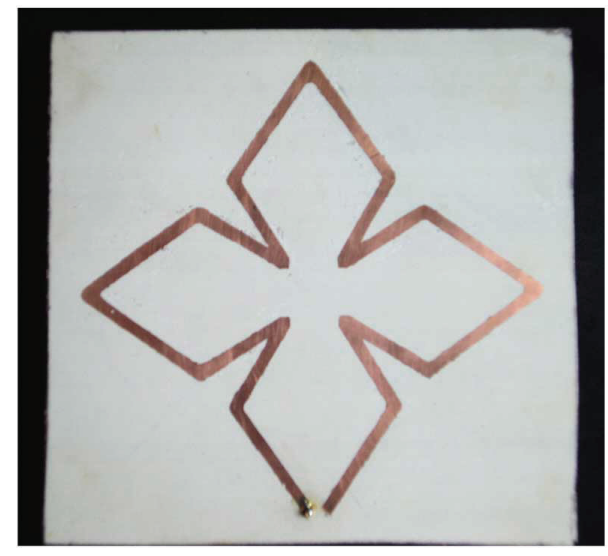

(b)

FIGURE 15: Constructed antennas (a) sharp edges and (b) soft edges.

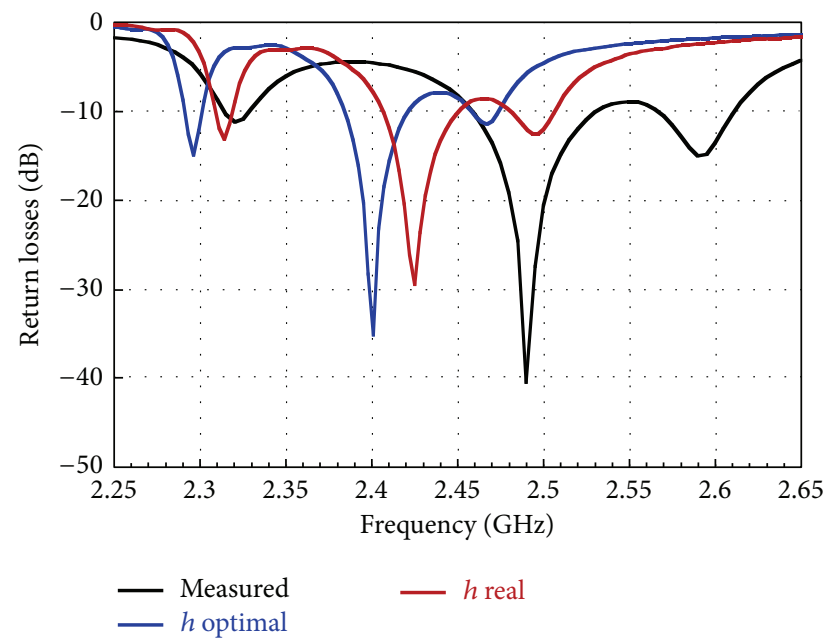

FIGURE 16: Return losses (antenna with sharp edges). 


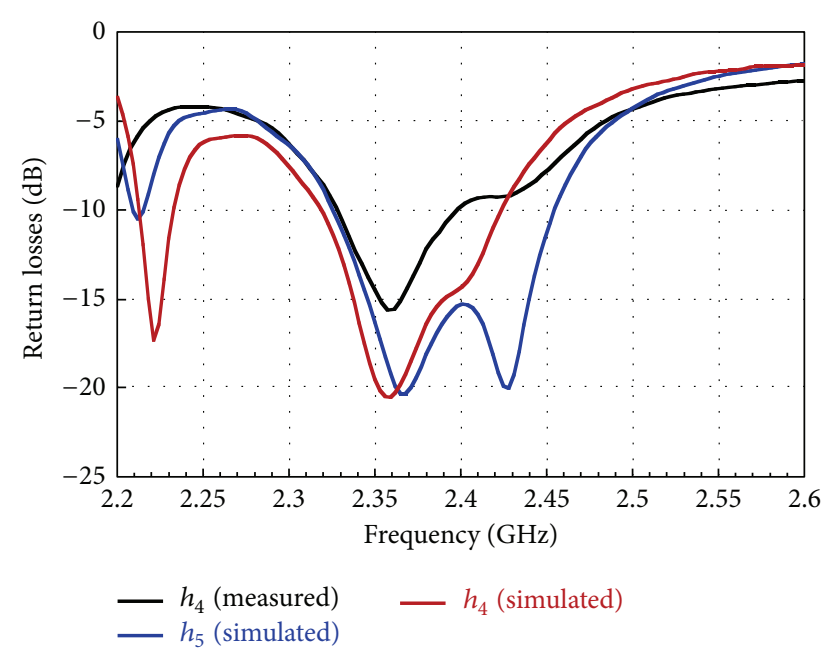

FIGURE 17: Return losses (antenna with softened edges).

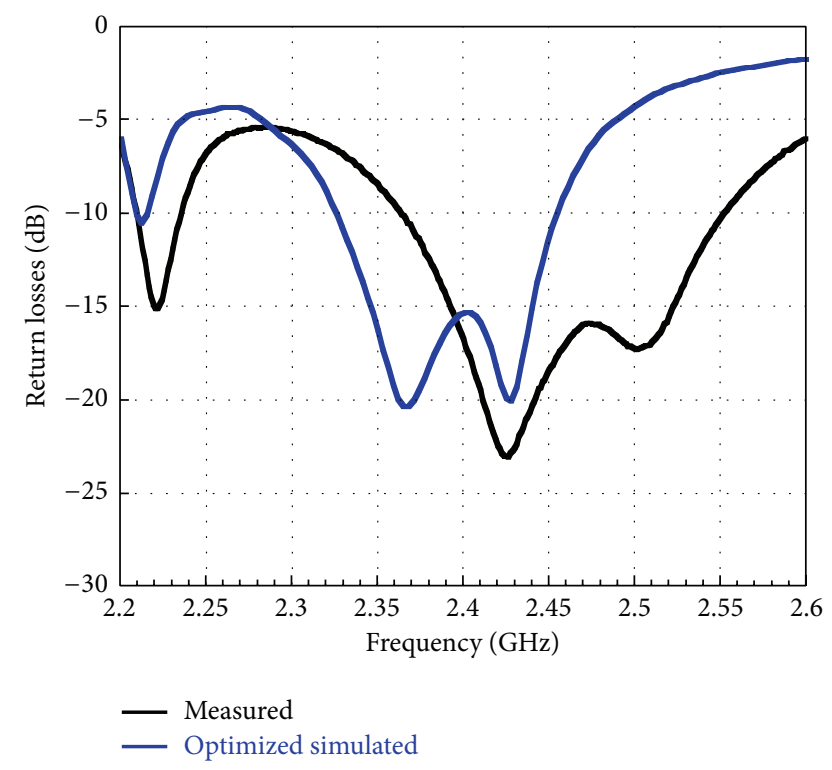

FIGURE 18: Measured return losses (CRA with softened edges).

good similarity between them but with a deviation of about $100 \mathrm{MHz}$ over design frequency.

On the other hand, Figure 17 shows comparison of $S_{11}$ parameters for same antennas but with softened edges; as it is seen, there is a good similitude between all of them. Finally, Figure 18 depicts coupling comparison between simulation and constructed softened edges antennas. We see again a deviation of about $70 \mathrm{MHz}$ between both antennas due to construction problems. Field patterns for constructed and simulation antennas are shown in Figure 19, also with some differences.

Figure 20 shows gain comparison of both antennas, and as seen, there are some differences, with a better response for the simulated one. It is clear that we have to improve our construction methods.

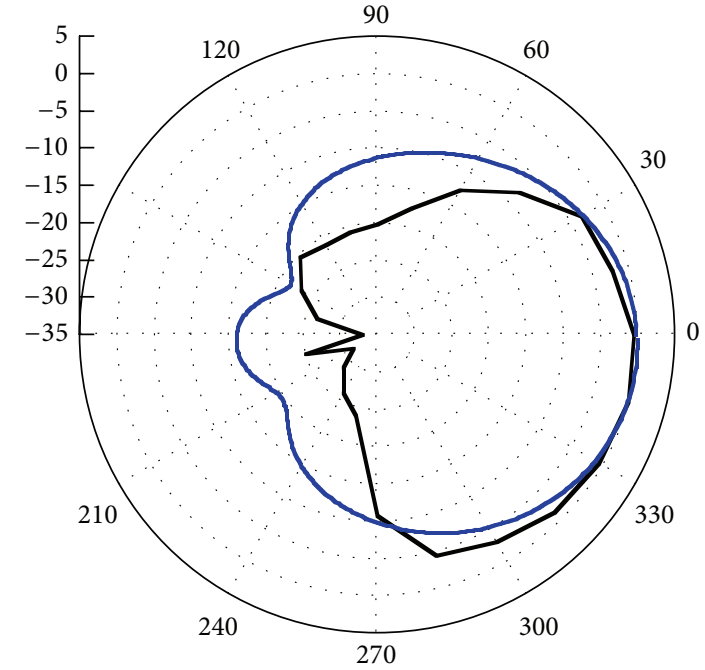

— Measured field pattern
- Simulated field pattern

FIGURE 19: Simulation and measured field pattern comparison.

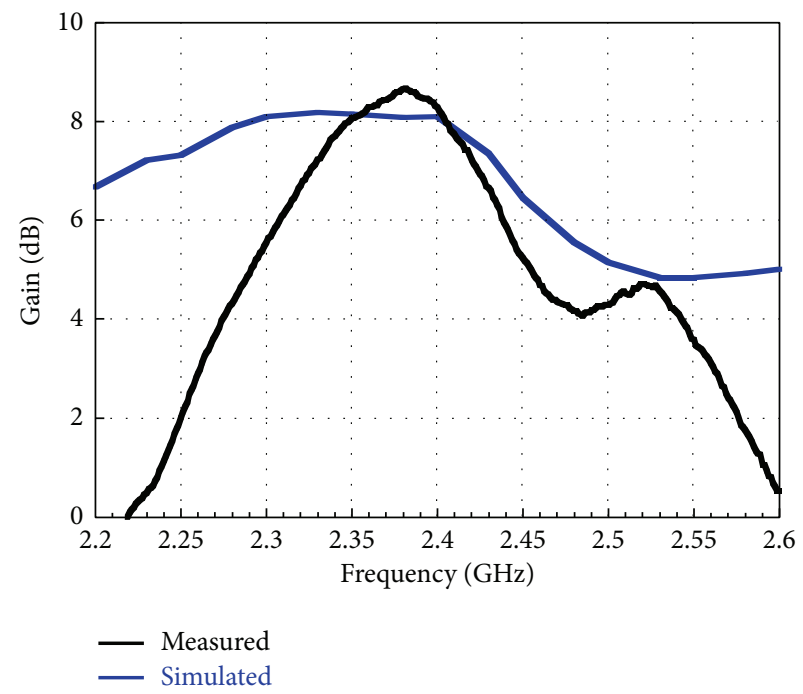

FiguRE 20: Gain comparison.

To characterize circular polarization, we obtained axial ratio, measuring horizontal and vertical field patterns. Both patterns have almost the same magnitude, except in $30^{\circ}$ and $285^{\circ}$, probing circular polarization. Figure 21 presents circular polarization measurements; (a) shows measured field patterns and (b) shows axial ratio obtained from subtraction magnitude of both field patterns.

\section{Conclusion}

We have presented parametric analysis of CRA with results of gain, field pattern, and return losses. Results shown of comparing simulation and constructed structures using RF60A with $\varepsilon_{r}=6.15$ agree with most of measured parameters 


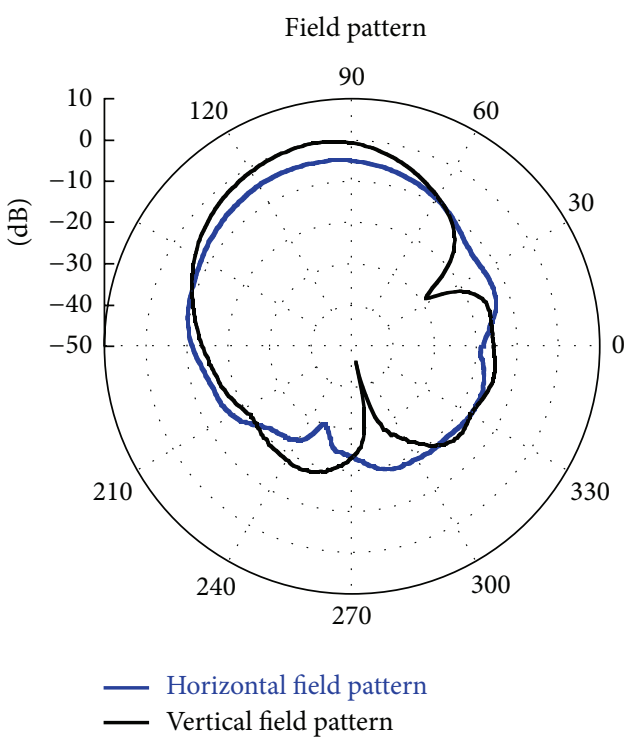

(a)

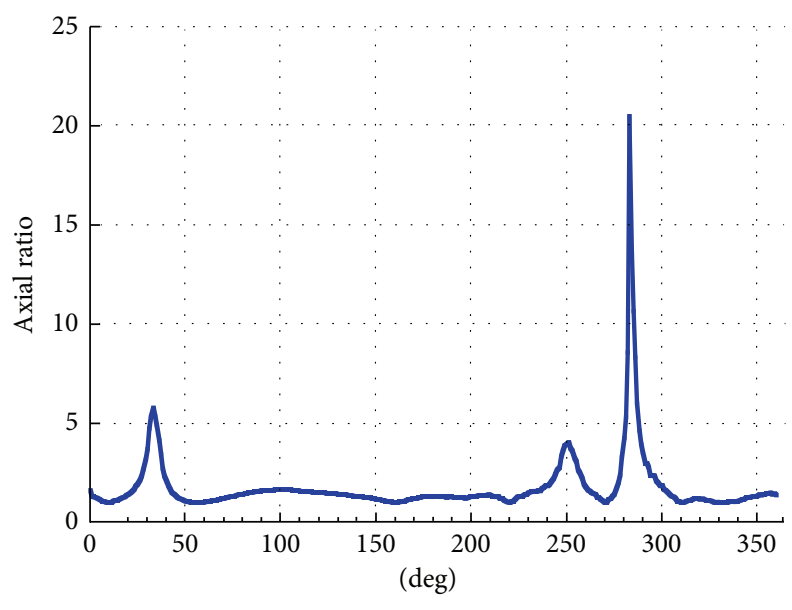

(b)

Figure 21: Circular polarization (a) field pattern comparison. (b) Axial ratio.

although with a displacement of about $100 \mathrm{MHz}$, meaning that error in the design frequency is due to manufacturing inaccuracy.

As it is seen from simulation, best configuration is that using open load, getting the best coupling and gain $(8.1 \mathrm{dBi}$ in the simulation and $7.9 \mathrm{dBi}$ in measurement). We also analyze the substrate thickness effects on resonant frequency.

A better similarity is obtained for the antenna with softening sharp edges considering its less dependence of frequency. We concluded that the best geometry for CRA is the one with soft edges with better performance and higher bandwidth.

We have proposed CRA for other applications, as those presented in $[6,7]$, where we designed and constructed air dielectric antennas for GNSS at $1.5 \mathrm{GHz}$ and also an air dielectric antenna for $2.4 \mathrm{GHz}$ with $14.0 \mathrm{dBi}$ gain and bandwidth around $60 \mathrm{MHz}$, for both of them, are greater than those of rectangular or circular patches presented in $[8,9]$.

The final dimensions of antenna are $10 \times 10 \times 0.56 \mathrm{~cm}$ with weight of $157 \mathrm{~g}$, and an excess of $1.6 \mathrm{~cm}$ over the $8.4 \mathrm{~cm}$ of maximum antenna dimension for the ground plane. Those $10 \mathrm{~cm}$ per side perfectly fit in the area of Sensat cube satellite.

\section{Conflict of Interests}

The authors declare that there is no conflict of interests.

\section{Acknowledgments}

The authors wish to thank to Instituto Politécnico Nacional and Consejo Nacional de Ciencia y Tecnología de México for their economic support.

\section{References}

[1] J. S. Pedroza, F. M. Zúñiga, and M. E. Aguilar, "Planar antennas for satellite communications," in Satellite Communications, N. Diodato, Ed., pp. 367-394, Sciyo, 2010.

[2] A. G. Roederer, "The cross antenna: a new low-profile circularly polarized radiator," IEEE Transactions on Antennas and Propagation, vol. 38, no. 5, pp. 704-710, 1990.

[3] E. G. Nolasco, Optimización de una antena plana para sistemas multiestándar [M.S. thesis], Sección de Estudios de Posgrado e Investigación, Escuela Superior de Ingeniería Mecánica y Eléctrica Zacatenco, IPN, Mexico City, Mexico, 2011.

[4] G. Kumar and K. Ray, Broadband Microstrip Antennas, Artech House, Norwood, Mass, USA, 2003.

[5] M. T. Ali, N. Ramli, M. K. M. Salleh, and M. N. M. Tan, "A design of reconfigurable rectangular microstrip slot patch antennas," in Proceedings of the IEEE International Conference on System Engineering and Technology (ICSET '11), pp. 111-115, Shah Alam, Malaysia, June 2011.

[6] J. S. Pedroza, L. E. C. Rivera, S. Peña Ruiz, and F. M. Zuñiga, "Análisis de acoplamiento mutuo en arreglos con antenas de cruz rómbica," in VI Conferencia Internacional en Ingeniería Electromecánica y de Sistemas (CIIES '11), November 2011.

[7] L. C. Rivera, J. S. Pedroza, S. P. Ruiz, and M. A. Mosqueda, "La técnica del patrón del elemento activo para analizar los efectos mutuos en un arreglo lineal de antenas Rómbicas de Cruz," in XXII Reunión Internacional de Otoño, de Comunicaciones, Computación, Electrónica, Automatización, Robótica y Aplicaciones Industriales y Exposición Industrial, IEEE, Guerrero, México, December 2011.

[8] H.-M. Chen, Y.-K. Wang, Y.-F. Lin, C.-Y. Lin, and S.-C. Pan, "Microstrip-fed circularly polarized square-ring patch antenna for GPS applications," IEEE Transactions on Antennas and Propagation, vol. 57, no. 4, pp. 1264-1267, 2009. 
[9] H. Li, J. Li, D. Li, and Y. Zhang, "High-gain circular polarization antenna for small satellite data link application," in Proceedings of the IEEE International Conference on Signal Processing, Communications and Computing (ICSPCC '11), Xian, China, September 2011. 

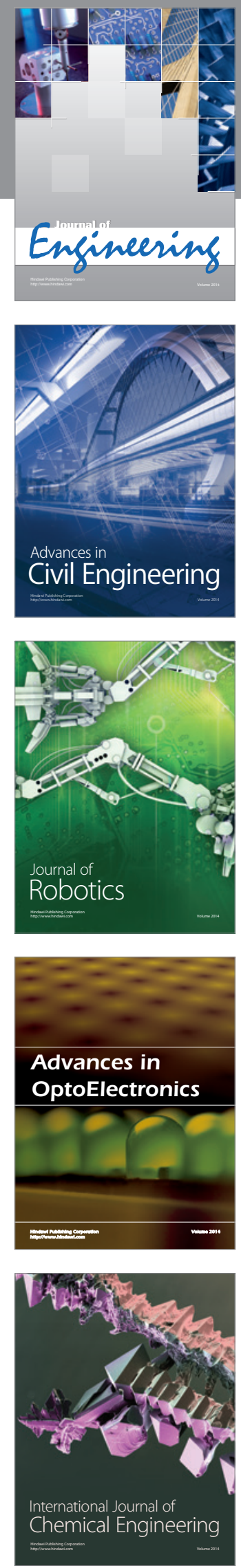

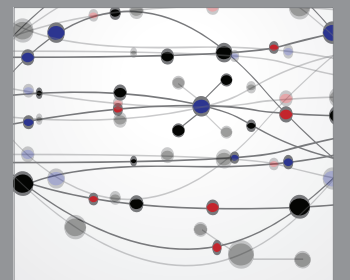

The Scientific World Journal
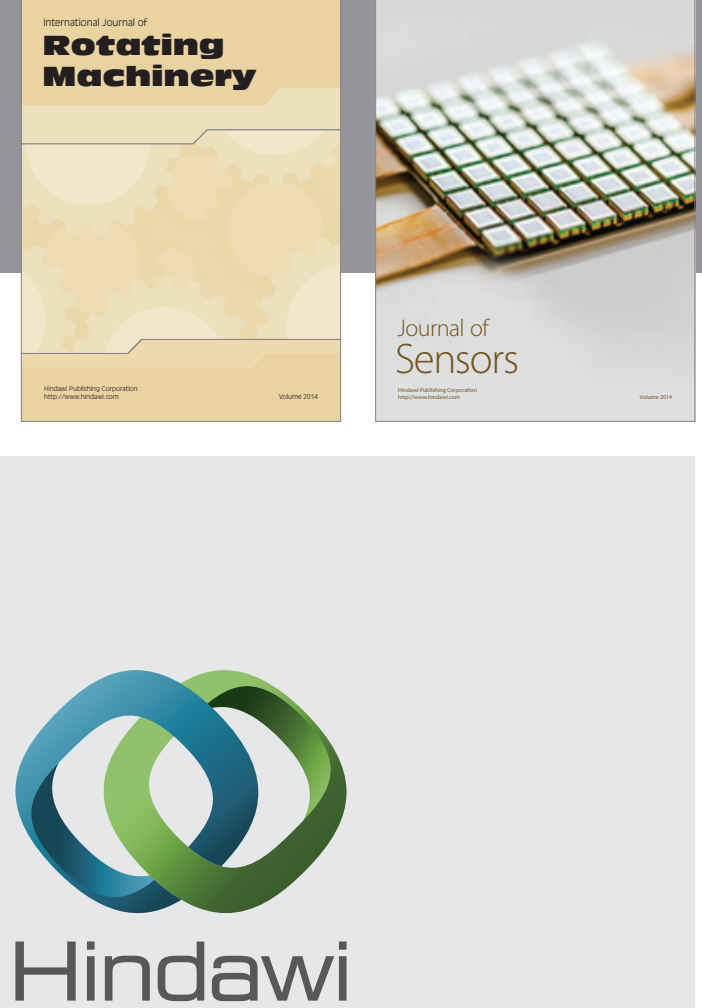

Submit your manuscripts at http://www.hindawi.com
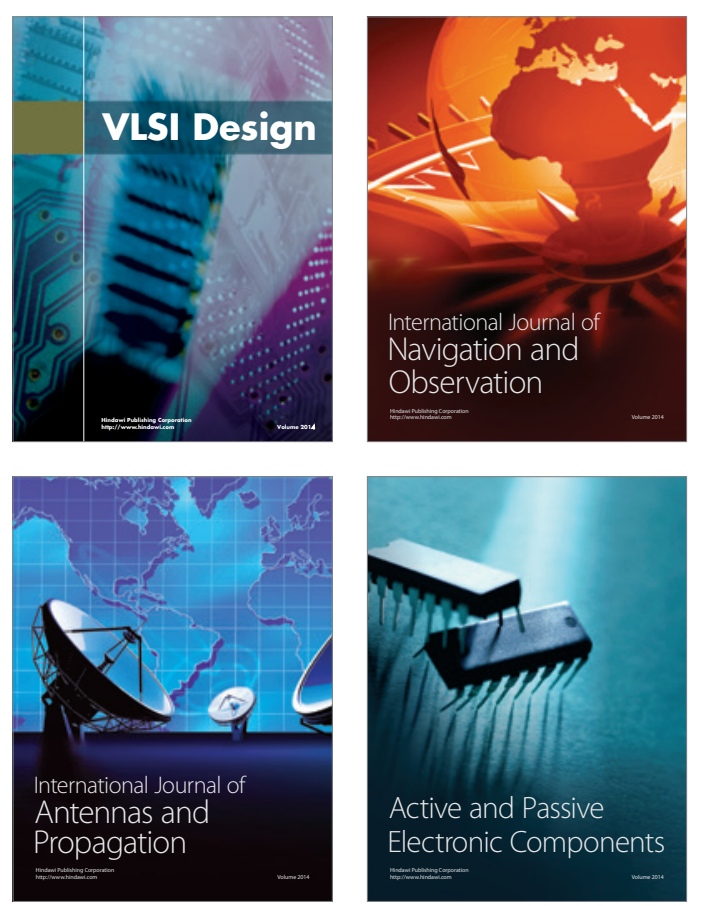
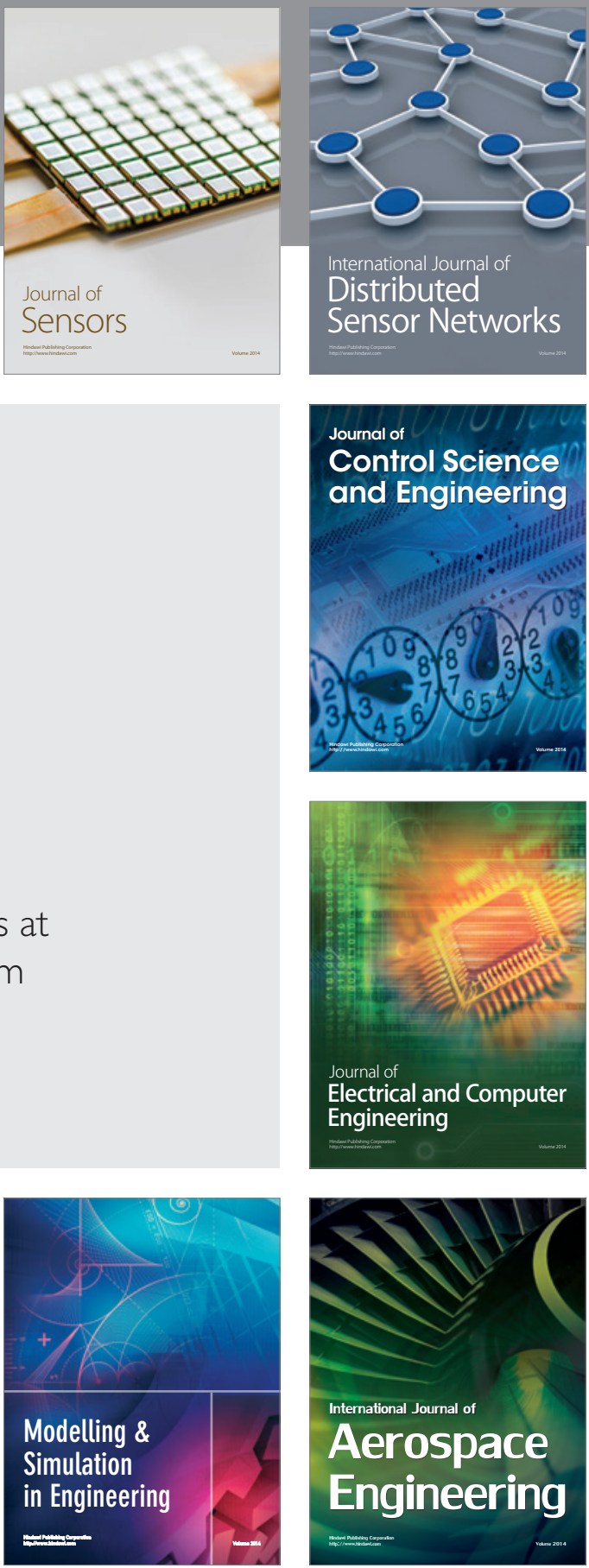

Journal of

Control Science

and Engineering
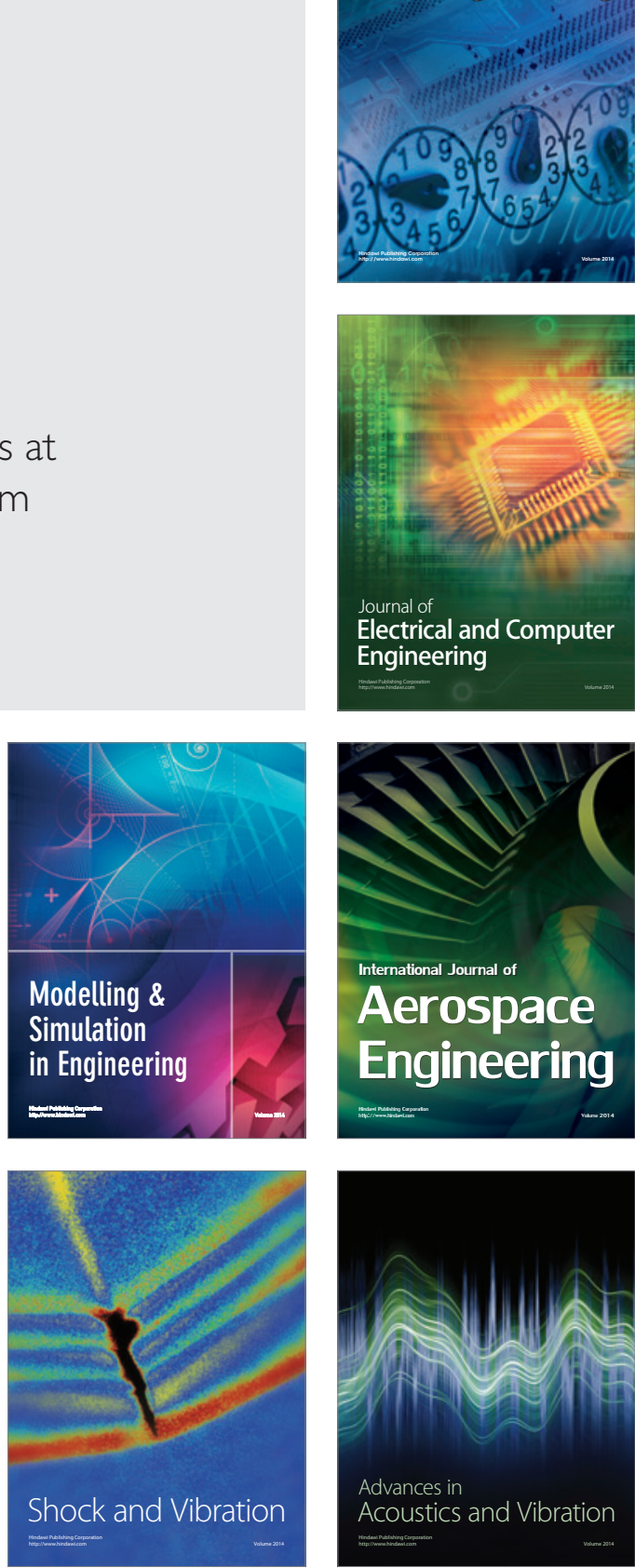